\title{
Modification of Social Dominance in Social Networks by Selective Adjustment of Interpersonal Weights
}

\author{
Mengbin $\mathrm{Ye}^{1}$, Ji Liu ${ }^{4}$, Brian D.O. Anderson ${ }^{1,2,3}$, Changbin Yu ${ }^{1,2}$, and Tamer Başar ${ }^{4}$
}

\begin{abstract}
According to the DeGroot-Friedkin model of a social network, an individual's social power evolves as the network discusses individual opinions over a sequence of issues. Under mild assumptions on the connectivity of the network, the social power of every individual converges to a constant nonnegative value as the number of issues discussed increases. If the network has a special topology, namely the "star topology", then all social power accumulates with the individual at the centre of the star. This paper studies the strategic introduction of new individuals and/or interpersonal relationships into a social network with the star topology so as to reduce the social power of the centre individual. In fact, several strategies are proposed. For each strategy, we derive necessary and sufficient conditions on the strength of the new interpersonal relationships, based on local information, which ensures that the centre individual no longer has the greatest social power within the social network. Interpretations of these conditions reveal that the strategies are remarkably intuitive and that certain strategies are favourable compared to others, all of which is sociologically expected.
\end{abstract}

\section{INTRODUCTION}

In recent years, the systems and control community has turned to study of networked systems and multi-agent systems in the context of social sciences. Of particular interest are social networks, where groups of people interact with acquaintances through interpersonal relationships.

One problem of "opinion dynamics" has been of particular interest: how do the opinions of individuals for a given issue evolve as they discuss this issue in a social network? A recent survey on opinion dynamics is presented in [1]. An important aspect is social power, which in one sense can be considered as the weight/power/influence an individual has on the opinion discussion, relative to the weight/power/influence of the other individuals in the social network. This relativity arises due to interpersonal relationships and their strengths (which may be unidirectional). This concept has been studied in the seminal works [2], [3]. The dynamical evolution of social power has been studied in [4]. Selecting the most

${ }^{1}$ M. Ye, B. D.O. Anderson and C. Yu are with the Research School of Engineering, Australian National University \{Mengbin.Ye, Brian.Anderson, Brad.Yu\}@anu.edu. au. ${ }^{2}$ B.D.O. Anderson and C. $\mathrm{Yu}$ are with Hangzhou Dianzi University, Hangzhou, China. ${ }^{3}$ B.D.O. Anderson is also with Data61-CSIRO (formerly NICTA Ltd.) in Canberra, A.C.T., Australia. ${ }^{4}$ J. Liu and T. Başar are with the Coordinated Science Laboratory, University of Illinois at Urbana-Champaign \{jiliu, basar1\}eillinois.edu. The work of Ye, Anderson, and $\mathrm{Yu}$ was supported by the Australian Research Council (ARC) under grants DP-130103610 and DP-160104500, by the National Natural Science Foundation of China (grant 61375072), and by Data61-CSIRO. Ye was supported by an Australian Government Research Training Program (RTP) Scholarship. The work of Liu and Başar was supported in part by Office of Naval Research (ONR) MURI Grant N00014-16-1-2710, and in part by NSF under grant CCF 11-11342. influential individual in social diffusion models has been studied in [5]. The centralised DeGroot-Friedkin model for the evolution of social power has been proposed and analysed in [6]. Distributed discrete- and continuous-time DeGrootFriedkin models have been proposed and studied in [7] and [8], respectively.

According to French Jr. and Snyder in [9], "leadership is the potential social influence of one part of the group over another." From the perspective of opinion dynamics, a leader can therefore be seen as an individual or a group of individuals that has a disproportionate amount of control over the opinion discussion process. In the context of social power, one can therefore refer to a leader/leader group as the socially dominant individual/group of individuals. The fact that social power tends to accumulate with one individual or a subgroup of individuals in a social network has been reported empirically in [4] and theoretically in [6]. This individual or subgroup is defined explicitly by the interpersonal relationships in the social network. Motivated by this concept of social dominance/leadership, and using the centralised DeGroot-Friedkin model to describe the social network, we begin in this paper with network topologies that have a single socially dominant individual, and seek to study strategies, including introduction of new individuals into the network and/or establishment of new interpersonal relationships, that will cause the social dominance to shift to another individual. The DeGroot-Friedkin model will be introduced formally in the sequel, but we describe it here at a high level so the reader is better able to understand the contributions of the paper. The DeGroot-Friedkin model considers a social network which discusses opinions of individuals on a sequence of issues/topics. At the end of the discussion of any given issue (which occurs when consensus on opinions is reached), each individual reflects on its influence, i.e. social power, on the overall discussion. For the following issue, each individual sets its self-weight (the weight the individual places on its own opinion relative to its neighbours' opinions) to be equal to its social power from the previous issue. The DeGrootFriedkin model describes the dynamical evolution of the social power of all individuals over the sequence of issues.

It was shown in [6] that if the social network has a constant topology, each individual's social power converges to a constant (equilibrium) value as issues were sequentially discussed. For the special star topology, all social power at equilibrium accumulated with a single individual $k$, in an "autocratic configuration". In this paper, we show that by strategic introduction of new individuals and/or new interpersonal relationships into the social network, not only is 
the autocratic configuration broken but if the new relationship is sufficiently strong, other identifiable individual(s) will have social power at equilibrium greater than individual $k$. Specifically, we derive necessary and sufficient conditions based on local information for the relationship strength. This is in contrast to many control strategies on networked systems which rely on global information [5], [10]. In fact, a number of different strategies are considered.

While the results are initially presented mathematically as inequalities, we provide detailed analysis and interpretation. In doing so, we show that the strategies are remarkably intuitive and precisely what one would expect when considered within a sociological context. While the strategies are intuitive, the novel contributions of this paper are the necessary and sufficient conditions for guaranteeing that the strategy succeeds, expressed as analytic functions of key interpersonal relationship strengths. The fact that the strategies affect the social power of individuals in a social network which is sequentially discussing issues implies that we have developed strategies for affecting/influencing the opinion dynamics process.

The rest of the paper is structured as follows. In Section II, we provide notations, an introduction to graph theory and the DeGroot-Friedkin model. At the same time, a formal problem statement is given. The main results are presented in Section III and conclusions are drawn in Section IV.

\section{BaCkground And Formal Problem Statement}

We begin by introducing some mathematical notations used in the paper. Let $\mathbf{1}_{n}$ and $\mathbf{0}_{n}$ denote, respectively, the $n \times 1$ column vectors of all ones and all zeros. For a vector $\boldsymbol{x} \in \mathbb{R}^{n}, 0 \preceq \boldsymbol{x}$ and $0 \prec \boldsymbol{x}$ indicate component-wise inequalities, i.e., for all $i \in\{1,2, \ldots, n\}, 0 \leq x_{i}$ and $0<x_{i}$, respectively. Let $\Delta_{n}$ denote the $n$-simplex, the set given by $\left\{\boldsymbol{x} \in \mathbb{R}^{n}: 0 \preceq \boldsymbol{x}, \mathbf{1}_{n}^{\top} \boldsymbol{x}=1\right\}$. The canonical basis of $\mathbb{R}^{n}$ is given by $\mathbf{e}_{1}, \ldots, \mathbf{e}_{n}$. Define $\widetilde{\Delta}_{n}=\Delta_{n} \backslash\left\{\mathbf{e}_{1}, \ldots, \mathbf{e}_{n}\right\}$ and $\operatorname{int}\left(\Delta_{n}\right)=\left\{\boldsymbol{x} \in \mathbb{R}^{n}: 0 \prec \boldsymbol{x}, \mathbf{1}_{n}^{\top} \boldsymbol{x}=1\right\}$. Note that the terms "node", and "individual" are used interchangeably.

\section{A. Graph Theory}

The interaction between individuals in a social network is modelled using a weighted directed graph, denoted as $\mathcal{G}=(\mathcal{V}, \mathcal{E})$. Each individual is a node in the finite, nonempty set of nodes $V=\left\{v_{1}, \ldots, v_{n}\right\}$. The set of ordered edges is $\mathcal{E} \subseteq \mathcal{V} \times \mathcal{V}$. We denote an ordered edge as $e_{i j}=\left(v_{i}, v_{j}\right) \in \mathcal{E}$, and because the graph is directed, in general $e_{i j}$ and $e_{j i}$ may not both exist. An edge $e_{i j}$ is said to be outgoing with respect to $v_{i}$ and incoming with respect to $v_{j}$. The presence of an edge $e_{i j}$ connotes that individual $j$ learns of, and takes into account, the opinion value of individual $i$ when updating its own opinion. The relative interaction matrix $C \in \mathbb{R}^{n \times n}$ associated with $\mathcal{G}$ has nonnegative entries $c_{i j}$, termed "relative interpersonal weights" in [6]. The entries of $\boldsymbol{C}$ have properties such that $0<c_{i j} \leq 1 \Leftrightarrow e_{j i} \in \mathcal{E}$ and $c_{i j}=0$ otherwise. It is assumed that $c_{i i}=0$ (i.e. with no self-loops), and we impose the restriction that $\sum_{j=1}^{n} c_{i j}=1$ (i.e. that $C$ is a row-stochastic matrix).
A directed path is a sequence of edges of the form $\left(v_{p_{1}}, v_{p_{2}}\right),\left(v_{p_{2}}, v_{p_{3}}\right), \ldots$ where $v_{p_{i}} \in \mathcal{V}, e_{i j} \in \mathcal{E}$. Node $i$ is reachable from node $j$ if there exists a directed path from $v_{j}$ to $v_{i}$. A graph is said to be strongly connected if every node is reachable from every other node. The relative interaction matrix $\boldsymbol{C}$ is irreducible if and only if the associated graph $\mathcal{G}$ is strongly connected. If $\boldsymbol{C}$ is irreducible then it has a unique left eigenvector $\gamma^{\top}$ satisfying $\gamma^{\top} \mathbf{1}_{n}=1$, with all entries strictly positive, associated with the eigenvalue 1 (PerronFrobenius Theorem, see [11]). Henceforth, we shall call $\gamma^{\top}$ the dominant left eigenvector of $\boldsymbol{C}$, with $i^{\text {th }}$ entry $\gamma_{i}$.

\section{B. The DeGroot-Friedkin Model}

We define $\mathcal{S}=\{0,1,2, \ldots\}$ to be the set of indices of sequential issues which are being discussed by the social network. For a given issue $s \in \mathcal{S}$, the social network's opinion dynamics process is described using the discretetime DeGroot opinion pooling algorithm (with constant weights throughout the discussion of the issue). At the end of the discussion (i.e. when the DeGroot model has effectively reached steady state), each individual reflects upon, and judges its impact on the discussion. This mechanism is termed reflected self-appraisal, with "reflection" referring to the fact that adjustments to weights are made following the completion of discussion on the particular issue $s$. The individual then updates its own self-weight (the weight an individual applies to its own opinion value in the DeGroot algorithm) and discussion begins on the next issue $s+1$ (using the same DeGroot algorithm but now with adjusted weights). We now explain the mathematical modelling of the opinion dynamics for an issue, and the updating of selfweights from one issue to the next.

For each issue $s \in \mathcal{S}$, individual $i$ updates its opinion $y_{i}(s, \cdot) \in \mathbb{R}$ at time $t+1$ as

$$
y_{i}(s, t+1)=w_{i i}(s) y_{i}(s, t)+\sum_{j \neq i}^{n} w_{i j}(s) y_{j}(s, t)
$$

where $w_{i i}(s)$ is the self-weight individual $i$ places on its own opinion and $w_{i j}(s)$ is the weight given by individual $i$ to the opinion of its neighbour individual $j$. Note that $w_{i j}(s), \forall i, j$ is constant for any given $s$. The element $w_{i j}, j \neq i$ is defined as $w_{i j}=\left(1-w_{i i}\right) c_{i j}$ which ensures that $\sum_{j=1}^{n} w_{i j}=1$ for any $w_{i i}$, because we have assumed above that $\sum_{j=1}^{n} c_{i j}=$ 1. In other words, $\sum_{j=1}^{n} w_{i j}(s)=1$ continues to hold as $w_{i i}(s)$ is updated (the precise mechanism for updating $w_{i i}(s)$ will be detailed shortly). It was shown in [6] that if $\boldsymbol{C}$ is irreducible, and $\exists i: w_{i i}>0$ then consensus of opinions is reached: $y_{k}(\infty)=y_{j}(\infty), \forall k, j$.

We now detail the updating of the self-weight (also referred to as individual social power, self-confidence or self-esteem) of individual $i$, which is denoted by $x_{i}(s)=$ $w_{i i}(s) \in[0,1]$ for convenience [6]. Define the vector $\boldsymbol{x}(s)=$ $\left[\begin{array}{lll}x_{1}(s) & \cdots & x_{n}(s)\end{array}\right]^{\top}$ as the vector of self-weights for the individuals of the social network, with starting self-weight $0 \leq x_{i}(0) \leq 1$ satisfying $\sum_{i} x_{i}(0)=1$. The DeGrootFriedkin model states that the self-weight vector $\boldsymbol{x}(s)$ is 
updated as

$$
\boldsymbol{x}(s+1)=\boldsymbol{F}(\boldsymbol{x}(s))
$$

where $\boldsymbol{F}(\boldsymbol{x}(s))$ is defined as

$$
\boldsymbol{F}(\boldsymbol{x}(s))= \begin{cases}\mathbf{e}_{i} & \text { if } x_{i}(s)=\mathbf{e}_{i}, \text { for any } i \\
\alpha(\boldsymbol{x}(s))\left[\begin{array}{c}
\frac{\gamma_{1}}{1-x_{1}(s)} \\
\vdots \\
\frac{\gamma_{n}}{1-x_{n}(s)}
\end{array}\right] & \text { otherwise }\end{cases}
$$

with $\alpha(\boldsymbol{x}(s))=1 / \sum_{i=1}^{n} \frac{\gamma_{i}}{1-x_{i}(s)}$. We omit the details on the precise formulation of the model, and the derivation of the map $\boldsymbol{F}$ due to space limitations; we refer the reader to the original work [6] which introduces the model.

Much of this paper will deal with scenarios where the underlying graph has a star topology or its variants, the definition of which is now given.

Definition 1 (Star topology). A strongly connected graph ${ }^{1} \mathcal{G}$ is said to have star topology if there exists a node $i$, which is called the centre node, such that every edge of $\mathcal{G}$ is either to or from node $i$

Note that the irreducibility of $C$ implies that the star topology must include edges in both directions between the centre node $v_{i}$ and every other node $v_{j}, j \neq i$. We now provide two results regarding the convergence of $\boldsymbol{F}(\boldsymbol{x}(s))$ as $s \rightarrow \infty$, with an interpretation given in Remark 1 .

Lemma 1 (Lemma 3.2, [6]). Suppose that $n \geq 3$, and suppose further that $\mathcal{G}$ has star topology, which without loss of generality has centre node $v_{1}$. Let $C$ be the rowstochastic and irreducible relative interaction matrix, with zero diagonal entries, associated with $\mathcal{G}$. Then for all initial conditions $\boldsymbol{x}(0) \in \widetilde{\Delta}_{n}, \lim _{s \rightarrow \infty} \boldsymbol{x}(s)=\mathbf{e}_{1}$.

Theorem 1 (Theorem 4.1, [6]). For $n \geq 3$, consider the DeGroot-Friedkin dynamical system (2) with a relative interaction matrix $\boldsymbol{C}$ that is row-stochastic, irreducible, and has zero diagonal entries. Assume that the digraph $\mathcal{G}$ associated with $C$ does not have star topology and define $\gamma^{\top}$ as the dominant left eigenvector of $\boldsymbol{C}$. Then,

(i) For all initial conditions $\boldsymbol{x}(0) \in \widetilde{\Delta}_{n}$, the self-weights $\boldsymbol{x}(s)$ converge to $\boldsymbol{x}^{*}$ as $s \rightarrow \infty$. Here, $\boldsymbol{x}^{*} \in \operatorname{int}\left(\Delta_{n}\right)$ is the unique fixed point satisfying $\boldsymbol{x}^{*}=\boldsymbol{F}\left(\boldsymbol{x}^{*}\right)$.

(ii) There holds $x_{i}^{*}<x_{j}^{*}$ if and only if $\gamma_{i}<\gamma_{j}$, for any $i, j$, where $\gamma_{i}$ is the $i^{\text {th }}$ entry of the dominant left eigenvector $\gamma$. There holds $x_{i}^{*}=x_{j}^{*}$ if and only if $\gamma_{i}=\gamma_{j}$.

(iii) The unique fixed point $x^{*}$ is determined only by $\gamma^{\top}$, and is independent of the initial conditions.

\section{Formal Problem Statement}

In this paper, we investigate how additional nodes and/or edges strategically connected to a star topology can change the social power at equilibrium, $\boldsymbol{x}^{*}$. To that end, we begin

\footnotetext{
${ }^{1}$ While it is possible to have a star graph that is not strongly connected, this paper, similarly to [6], deals only with strongly connected graphs.
}

first by providing definitions which will aid in describing our problem and discussing the results obtained. Moreover, we are interested in comparing the social power of individuals within the network at equilibrium, i.e. when $s \rightarrow \infty$. We will therefore refer to the equilibrium value $x_{i}^{*}$ as the social power of individual $i$ when there is no ambiguity (as opposed to the evolving $x_{i}(s)$ when $\left.s<\infty\right)$. To simplify the problem, we do not study the evolution of the opinions $y_{i}(s, t)$, which as observed below (1), will always reach a consensus if $\boldsymbol{C}$ is irreducible and $\boldsymbol{x}(0) \in \Delta_{n}$.

Definition 2 (Autocratic Network). A social network is said to be an autocratic configuration, with node $v_{i}$ being the autocrat, if $\boldsymbol{x}(s)=\mathbf{e}_{i}$.

Definition 3 (Social dominance/leadership). Node $v_{i}$ is said to be the socially dominant/leader node in the network if $x_{i}^{*}>x_{j}^{*}$ for all $j \neq i$. In other words, at equilibrium, the social power of individual $i$ is greater than the social power of any other individual in the social network.

Remark 1 (Autocratic tendency). Lemma 1 has an important social connotation. One can consider $x_{i}(0)$ as individual $i$ 's estimate of its social power when the social network is first formed, before any discussions occur. For any initial estimate $\boldsymbol{x}(0) \in \widetilde{\Delta}_{n}$ (that is, no individual $i$ believes $x_{i}(0)=1$ ), the star topology network tends to an autocratic configuration at equilibrium, $\boldsymbol{x}^{*}=\mathbf{e}_{1}$. This implies that, for initial issues, opinion discussion will occur with everyone contributing to the final consensus value. However, the centre individual increasingly guides the outcome of discussions until, for $s=\infty$, only the centre individual's opinion value matters.

Remark 2. In [6], the constant entries $c_{i j}$ of $\boldsymbol{C}$ are termed "relative interpersonal weights", and we will stay with this terminology. However, one can also consider $c_{i j}$ as the amount of relative "trust" individual i allocates to individual $j$. In other words, $c_{i j}$ captures the strength of a unidirectional interpersonal relationship (unidirectional since $c_{i j} \neq c_{j i}$ in general).

For a given graph $\mathcal{G}$ with star topology, with centre node $v_{1}$, let us call the other nodes subject nodes in the sense that they are subjects to the autocrat centre node. We are going to study how the autocracy can be disrupted by introduction of a perturbation to the star graph. This leads us to define a new type of node. An attacker node is a node $v_{j}$ which forms edges $e_{j i}, e_{i j}$ with a subject node. In doing so, we modify the graph $\mathcal{G}$ to become $\overline{\mathcal{G}}$ which is no longer a star. In Fig. 1 , $v_{1}$ is the centre node, while nodes $v_{i}, i=2, \ldots, 7$ are subject nodes, and node $v_{8}$ is the attacker node, forming edges with node $v_{7}$. We call node $v_{j}$ an attacker node because, as it will become apparent in the sequel, the weights $c_{j i}$ and $c_{i j}$ can modify/reduce the social power $x_{1}^{*}$ of the centre node $v_{1}$. In other words, $v_{j}$ attacks the social dominance of $v_{1}$. Note that two edges, $e_{j i}, e_{i j}$ must be formed to ensure that $\overline{\mathcal{G}}$ remains strongly connected. Actually, there are a number of interesting ways to attack the social dominance of $v_{1}$, and we list below some of the most important/fundamental methods. 
An example of each is given in Figures 1-4.

Topology Variation 1 (Single Attack). Suppose that $n \geq 4$. Suppose further that $\mathcal{G}$ has star topology, with $v_{1}$ being the centre node, and with $n-2$ subject nodes, $v_{i}, i=2, \ldots, n-1$. A single attacker node $v_{n}$ attaches to subject node $v_{n-1}$ by forming edges $e_{n-1, n}, e_{n, n-1}$, forming the graph $\overline{\mathcal{G}}$.

Topology Variation 2 (Coordinated Double Attack). Suppose that $n \geq 5$. Suppose further that $\mathcal{G}$ has star topology, with $v_{1}$ being the centre node, and with $n-3$ subject nodes, $v_{i}, i=2, \ldots, n-2$. Two attacker nodes $v_{n-1}$ and $v_{n}$ attach to subject node $v_{n-2}$ by forming the set of edges $\left\{e_{n-2, n-1}, e_{n-1, n-2}, e_{n-2, n}, e_{n, n-2}\right\}$. This forms $\overline{\mathcal{G}}$.

Topology Variation 3 (Uncoordinated Double Attack). Suppose that $n \geq 5$. Suppose further that $\mathcal{G}$ has star topology, with $v_{1}$ being the centre node, and with $n-3$ subject nodes, $v_{i}, i=2, \ldots, n-2$. One attacker node $v_{n-1}$ attaches to subject node $v_{n-3}$ with edges $e_{n-3, n-1}, e_{n-1, n-3}$. A second attacker node $v_{n}$ attaches to subject node $v_{n-2}$ with edges $e_{n-2, n}, e_{n, n-2}$. This forms $\overline{\mathcal{G}}$.

Topology Variation 4 (Two Dissenting Subjects). Suppose that $n \geq 4$. Suppose further that $\mathcal{G}$ has star topology, with $v_{1}$ being the centre node, and with $n-1$ subject nodes, $v_{i}, i=2, \ldots, n$. There are no attacker nodes. Subject nodes $v_{n-1}$ and $v_{n}$ form edges $e_{n, n-1}, e_{n-1, n}$, forming $\overline{\mathcal{G}}$.

In the next section, we investigate the above topological variations of the star graph. Note that Topology Variations 14 have modified graphs $\overline{\mathcal{G}}$ which do not have star topology. From Theorem 1, it immediately follows that $x_{1}^{*}<1$ for all Topology Variations. In other words, $v_{1}$ is no longer the autocrat but if the perturbation from the star topology (caused by the new edges) is small, $v_{1}$ remains socially dominant. What we will show is that if the interpersonal weights associated with these new edges exceed a given threshold, the socially dominant node changes from $v_{1}$ to some other node. Of particular note is that the thresholds depend only on local information. It is worth emphasising at this stage that, in Variations 1-3, it is useless for an attacker node $v_{n}$ to attach to the centre node $v_{1}$ instead of a subject node; the topology remains a strongly connected star, and thus $v_{1}$ remains the socially dominant autocrat.

Note that when new edges are introduced, we assume each individual $i$ adjusts its weights $c_{i j}$ to ensure that the new $\boldsymbol{C}$ is row-stochastic. Take Topology Variation 4 as an example. The relative interaction matrix $\boldsymbol{C}$, associated with star graph $\mathcal{G}$ (without edges $e_{n, n-1}$ and $e_{n-1, n}$ ), is assumed to be rowstochastic. The relative interaction matrix $\bar{C}$ associated with $\overline{\mathcal{G}}$ is also implicitly assumed to be row-stochastic with zero diagonal. That is, after the addition of edges $e_{n, n-1}, e_{n-1, n}$, adjustments are made to the original weights $c_{n, j}, c_{n-1, k}$ to ensure that $\bar{C}$ is row-stochastic.

Remark 3 (Ordering of Social Power). Although Theorem 1 states that $\boldsymbol{x}^{*}$ is uniquely determined by $\gamma^{\top}$, there are no results available which allow one to analytically compute the value of $\boldsymbol{x}^{*}$ given $\boldsymbol{\gamma}^{\top}$. What is available is Statement (ii) of
Theorem 1, which states that the ordering of $\boldsymbol{x}_{i}^{*}$ is consistent with the ordering of $\gamma_{i}$. A recent paper [12] computed an upper bound on $x_{i}^{*}$ which is dependent on $\gamma_{i}$. However, we are in this paper interested in the ordering of individual social power, as opposed to the precise values of social power. This is reflected in Definition 3.

\section{Main Results}

In order to place the focus on discussion of the social connotations of each result, and due to space limitations, the proofs of the results presented in this section are not included in this paper. The proofs for each theorem and corollary, which all follow the same format, can be found in the extended version of this paper on arXiv [13]. Simulations demonstrating our main results are also provide in [13].

Topology Variation 1 is in fact a special case of Topology Variation 2, obtained by setting $c_{n, n-2}=0$ and by removing node $v_{n}$. As such, the first result we present will be on Variation 2, the discussions on Variation 2 are all applicable to the fundamental strategy Variation 1 with minor adjustments.

\section{A. Topology Variation 2: Coordinated Double Attack}

Consider now Topology Variation 2. Firstly, define $\beta_{1}=$ $c_{n-2, n-1} \in(0,1)$ and $\beta_{2}=c_{n-2, n}=(0,1)$ as the two adjustable interpersonal weights associated with the two attackers. Note that because $\overline{\boldsymbol{C}}$ is assumed to be row-stochastic, it is implied that $\beta_{1}+\beta_{2}+c_{n-2,1}=1 \Rightarrow \beta_{1}+\beta_{2}<1$ because $c_{n-2,1}>0$. It is straightforward to observe that

$\overline{\boldsymbol{C}}\left(\beta_{1}, \beta_{2}\right)=\left[\begin{array}{cccccc}0 & c_{12} & \ldots & c_{1, n-1} & 0 & 0 \\ 1 & 0 & \ldots & 0 & 0 & 0 \\ \vdots & \vdots & \ddots & \vdots & \vdots & \vdots \\ 1-\left(\beta_{1}+\beta_{2}\right) & 0 & \ldots & 0 & \beta_{1} & \beta_{2} \\ 0 & 0 & \ldots & 1 & 0 & 0 \\ 0 & 0 & \ldots & 1 & 0 & 0\end{array}\right]$

We do not display the exact form of $\overline{\boldsymbol{C}}$ for other Topology Variations in this paper due to space limitations, but they are provided in the Appendix of [13].

Theorem 2 (Coordinated Double Attack). For a social network with Topology Variation 2, with initial conditions $\boldsymbol{x}(0) \in \widetilde{\Delta}_{n}$, and described by the DeGroot-Friedkin model, the following statements are true:

(i) For all $\beta_{1}, \beta_{2} \in(0,1)$, there holds $x_{i}^{*}<x_{1}^{*}$ for all $i \neq 1, n-2, n-1, n$, and $x_{n-1}^{*}, x_{n-1}^{*}<x_{n-2}^{*}$.

(ii) There holds 1) $x_{1}^{*}>x_{i}^{*}, \forall, i \neq 1$ if and only if $\beta_{1}+\beta_{2}<$ $1-c_{1, n-2}=\sum_{i=2}^{n-3} c_{1, i}$, or 2) $x_{n-2}^{*}>x_{i}^{*}, \forall, i \neq n-2$ if and only if $\beta_{1}+\beta_{2}>1-c_{1, n-2}$. There holds $x_{1}^{*}=x_{n-2}^{*}$ if and only if $\beta_{1}+\beta_{2}=1-c_{1, n-2}$.

(iii) There holds $x_{n}^{*}>x_{1}^{*}$ (respectively $x_{n-1}^{*}>x_{1}^{*}$ ) if and only if $\beta_{2}>\left(1-\beta_{1}\right) /\left(1+c_{1, n-2}\right)$ (respectively $\beta_{1}>$ $\left.\left(1-\beta_{2}\right) /\left(1+c_{1, n-2}\right)\right)$.

(iv) There holds $x_{n-1}^{*}<x_{n}^{*}$ or $x_{n-1}^{*}>x_{n}^{*}$ if and only if $\beta_{1}<\beta_{2}$ or $\beta_{1}>\beta_{2}$ respectively. If $\beta_{1}=\beta_{2}$, then $x_{n-1}^{*}=x_{n}^{*}$.

Corollary 1 (Generalised Placement of Coordinated Double Attack). Suppose that instead of attaching to subject node 


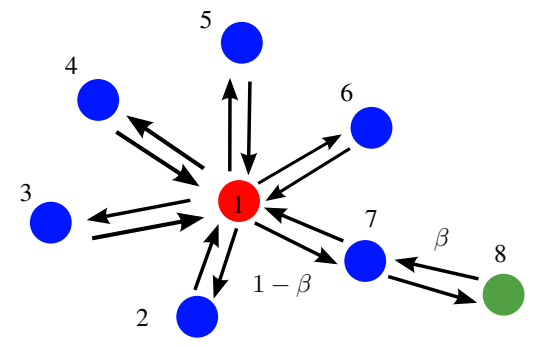

Fig. 1. Topology Variation 1 (Single Attacker) with $n=8$, attacker is green.

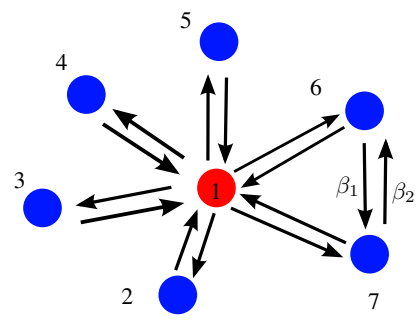

Fig. 4. Topology Variation 4 (Two Dissenting Subjects) with $n=7$.

$v_{n-2}$, attacker nodes $v_{n-1}, v_{n}$ can attach to any subject node $v_{i}, i \in\{2, \ldots, n-2\}$ by forming the set of edges $\left\{e_{n-1, i}, e_{i, n-1}, e_{n, i}, e_{i, n}\right\}$. The lower bound on $\beta_{1}+\beta_{2}=$ $c_{n-1, i}+c_{n, i}$ required to have $x_{n-2}^{*}>x_{1}^{*}$ is minimised if $v_{n-1}$ and $v_{n}$ attach to $v_{k}$ where $k=\operatorname{argmax}_{j \in\{2, \ldots, n-2\}} c_{1, j}$.

The above mathematical results can be interpreted in the following social context. From Statement (i), we conclude that subject individual $i$, for $i \in\{2, . ., n-3\}$, will never have greater social power at equilibrium than the centre individual $v_{1}, x_{i}^{*}<x_{1}^{*}$, regardless of how $\beta_{1}, \beta_{2}$ changes. Moreover, the attacker nodes $v_{n-1}, v_{n}$ will never have greater social power than the subject node $v_{n-2}$ which it is attached to.

Remark 2 states that $c_{i j}$ can be considered the trust level accorded to individual $j$ by individual $i$. The key result is Statement (ii), which indicates that the combined trust given to attackers $v_{n-1}$ and $v_{n}$ by subject node $v_{n-2}$ must exceed the combined trust given to subjects $v_{2}, \ldots, v_{n-3}$ by centre node $v_{1}$, in order for centre node $v_{1}$ to lose social dominance (and thus subject $v_{n-2}$ becomes socially dominant). It is most interesting to note that it is only the sum of the trust/influence $\beta_{1}+\beta_{2}$ that is relevant, and there is no requirement on the individual magnitudes of $\beta_{1}, \beta_{2}$.

Corollary 1 delivers an intuitive, powerful, and socially relevant result. It states that the attackers $v_{n-1}, v_{n}$ should seek to form an interpersonal relationship with the subject individual $v_{k}$ that individual $v_{1}$ trusts the most. This will minimise the required amount of trust subject $v_{k}$ accords attacker $v_{n}$ before centre individual $v_{1}$ loses social dominance.

Statement (iii) reveals that the attackers can also obtain social power greater than the centre individual $v_{1}$ if $\beta_{1}$ and $\beta_{2}$ exceed some lower bounding inequalities. Specifically, we observe that the inequality, which if satisfied ensures that attacker $v_{n}$ has social power greater than centre $v_{1}$,

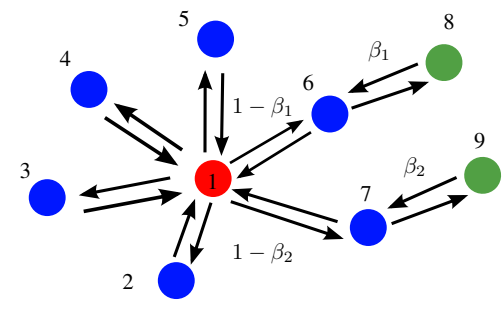

Fig. 3. Topology Variation 3 (Uncoordinated Double Attacker) with $n=9$, green attackers.

simply involves the variables $\beta_{1}, \beta_{2}$ and $c_{1, n-2}$. Moreover, there always exists a pair $\beta_{1}, \beta_{2}$, satisfying $\beta_{1}+\beta_{2}<1$ (and thus maintaining $\sum_{j=1}^{n} c_{n-2, j}=1$ ), which ensures both attacker individuals $v_{n-1}, v_{n}$ have social power greater than the centre $v_{1}$. This is detailed in the proof [13, Theorem 2] and is desirable from the view point of the two attacker individuals.

\section{B. Topology Variation 3: Uncoordinated Double Attack}

Define $\beta_{1}=c_{n-3, n-1} \in(0,1)$ and $\beta_{2}=c_{n-2, n} \in(0,1)$.

Theorem 3 (Uncoordinated Double Attack). For a social network with Topology Variation 3, with initial conditions $\boldsymbol{x}(0) \in \widetilde{\Delta}_{n}$, and described by the DeGroot-Friedkin model, the following statements are true:

(i) For all $\beta_{1}, \beta_{2} \in(0,1)$, there holds $x_{i}^{*}<x_{1}^{*}$ for all $i \in\{2, \ldots, n-4\}$, and $x_{n-1}^{*}<x_{n-3}^{*}$ and $x_{n}^{*}<x_{n-2}^{*}$.

(ii) There holds $x_{1}^{*}>x_{i}^{*}$ for all $i \neq 1$ if and only if $\beta_{1}<1-c_{1, n-3}$ and $\beta_{2}<1-c_{1, n-2}$. If $\beta_{1}>1-c_{1, n-3}$ (respectively $\beta_{2}>1-c_{1, n-2}$ ), then $x_{n-3}^{*}>x_{1}^{*}$ (respectively $x_{n-2}^{*}>x_{1}^{*}$ ).

(iii) For $i \in\{1,2\}$, there holds $x_{n-2+i}^{*}>x_{1}^{*}$ if and only if $\beta_{i}>1 /\left(1+c_{1, n-4+i}\right)$.

(iv) There holds $x_{n-3}^{*}>x_{n-2}^{*}$ if and only if $\frac{1-\beta_{2}}{1-\beta_{1}}>\frac{c_{1, n-2}}{c_{1, n-3}} \Leftrightarrow \frac{c_{1, n-3}}{c_{n-3,1}}>\frac{c_{1, n-2}}{c_{n-2,1}}$.

Due to space limitations, we discuss social implications of Theorem 3 only if the conclusions differ significantly from the discussion in the previous subsection.

The most interesting conclusion drawn from Theorem 3 arises when we compare to Theorem 2 which concerns Topology Variation 2. With Topology Variation 2, for the centre individual $v_{1}$ to lose its social dominance we require the sum of the trust values $\beta_{1}+\beta_{2}$ to exceed a lower bound, and there are no separate lower bounding inequalities for $\beta_{1}$ or $\beta_{2}$. With Topology Variation 3 , centre individual $v_{1}$ loses social dominance if and only if either $\beta_{1}$ or $\beta_{2}$ exceed their respective lower bounding inequalities. Importantly, these two lower bounding inequalities are independent of each other. This clearly points to the fact that a coordinated attack on the social dominance of the centre node is more desirable, an idea which is socially intuitive.

From Statement (iii), both attacker nodes have larger social power than the centre node if and only if $\beta_{1}>1 /(1+$ $\left.c_{1, n-3}\right)$ and $\beta_{2}>1 /\left(1+c_{1, n-2}\right)$, which implies that $\beta_{1}+$ $\beta_{2}>1 /\left(1+c_{1, n-3}\right)+1 /\left(1+c_{1, n-2}\right)$. From Statement (iii) 
Theorem 2, with Topology Variation 2, both attacker nodes have larger social power than the centre node if and only if $\beta_{2}>\left(1-\beta_{1}\right) /\left(1+c_{1, n-2}\right)$ and $\beta_{1}>\left(1-\beta_{2}\right) /\left(1+c_{1, n-2}\right)$, which implies that $\beta_{1}+\beta_{2}>2 /\left(2+c_{1, n-2}\right)$. Since both $1+$ $c_{1, n-2}$ and $1+c_{1, n-3}$ are smaller than $2+c_{1, n-2}$, it follows that $1 /\left(1+c_{1, n-3}\right)+1 /\left(1+c_{1, n-2}\right)>2 /\left(2+c_{1, n-2}\right)$, which implies that a coordinated attack on the social dominance of the centre node is also more efficient for the attackers.

\section{Topology Variation 4: Two Dissenting Subjects}

Topology Variation 4 is different from the ones studied above in the sense that there are no attacker nodes. Instead, one can consider this variation as one where two subjects form a relationship in dissent from the leader. Letting $\beta_{1}=$ $c_{n-1, n} \in(0,1)$ and $\beta_{2}=c_{n, n-1} \in(0,1)$, analysis yields:

Theorem 4 (Two Dissenting Subjects). For a social network with Topology Variation 4, with initial conditions $\boldsymbol{x}(0) \in \widetilde{\Delta}_{n}$, and described by the DeGroot-Friedkin model, the following statements are true:

(i) For all $\beta_{1}, \beta_{2} \in(0,1)$, there holds $x_{i}^{*}<x_{1}^{*}$ for all $i \neq 1, n-1, n$.

(ii) There holds $x_{n}^{*}>x_{1}^{*}$ if and only if $\beta_{1}>(1-$ $\left.c_{1, n}\right) /\left(c_{1, n-1}+\beta_{2}\right)$ with $\beta_{1} \in(0,1)$. There exists such $a \beta_{1} \in(0,1)$ only if $\beta_{2}>\sum_{i=2}^{n-2} c_{1, i}$.

(iii) There holds $x_{n-1}^{*}>x_{1}^{*}$ if and only if $\beta_{2}>(1-$ $\left.c_{1, n-1}\right) /\left(c_{1, n}+\beta_{1}\right)$ with $\beta_{2} \in(0,1)$. There exists such a $\beta_{2} \in(0,1)$ only if $\beta_{1}>\sum_{i=2}^{n-2} c_{1, i}$.

(iv) There holds $x_{n}^{*}<x_{n-1}^{*}$ if and only if $\beta_{2}>\beta_{1} c_{1, n}+$ $c_{1, n-1}\left(c_{1, n}-1\right)$ or equivalently $\beta_{1}<\left(\beta_{2}+c_{n-1}(1-\right.$ $\left.\left.c_{1, n}\right)\right)$

Note that the inequality in statement (ii) can be rewritten as $\beta_{2}>\left(1-c_{1, n}-\beta_{1} c_{1, n-1}\right) / \beta_{1}$ with $\beta_{2} \in(0,1)$ which is satisfiable only if $\beta_{1}>\left(1-c_{1, n}\right) /\left(1+c_{1, n-1}\right)$. Similarly, the inequality in statement (iii) is equivalent to $\beta_{1}>(1-$ $\left.c_{1, n-1}-\beta_{2} c_{1, n}\right) / \beta_{2}$ with $\beta_{1} \in(0,1)$ which is satisfiable only if $\beta_{2}>\left(1-c_{1, n-1}\right) /\left(1+c_{1, n}\right)$.

We now interpret Statement (ii), which we view, along with Statement (iii), as the key result of the theorem. A similar conclusion can be drawn for Statement (iii) but we do not include it due to space limitations. In order to make centre node $v_{1}$ lose social dominance, the dissent subject nodes $v_{n-1}$ and $v_{n}$ must adopt a cooperative strategy. From their definitions, we can interpret $\beta_{1}$ as the trust given by $v_{n-1}$ to $v_{n}$ while $\beta_{2}$ is the trust given by $v_{n}$ to $v_{n-1}$. A necessary condition for individual $v_{n}$ to have social power greater than centre node $v_{1}$ is that $\beta_{2}>\sum_{i=2}^{n-2} c_{1, i}$. This means that not only must $v_{n-1}$ trust $v_{n}$ sufficiently (as given by the inequality $\left.\beta_{1}>\left(1-c_{1, n}\right) /\left(c_{1, n-1}+\beta_{2}\right)\right)$, but individual $v_{n}$ must reciprocate by ensuring that it trusts $v_{n-1}$ sufficiently $\left(\beta_{1}>\sum_{i=2}^{n-2} c_{1, i}\right)$. Unless the two dissenting nodes build a cooperative and sufficiently strong bilateral relationship, centre node $v_{1}$ will remain socially dominant.

Remark 4. It should be noted that we have analysed the strategies and discussed results and implications from the perspective of attacker and subject nodes. It would be of great interest in future work to analyse from the leader's perspective and consider how to ensure robustness and protection against external influence. This also opens up future work using a game theoretic approach.

\section{CONCLUSIONS}

Social networks with the star topology converge to an autocratic configuration, with the centre individual holding all the social power. This paper proposed a number of different strategies, involving introduction of new individuals and/or new interpersonal relationships into the social network, in order to move social dominance from the centre individual to a subject individual. Necessary and sufficient conditions have been developed, and based on these conditions it has been argued that the strategies are sociologically intuitive. $\mathrm{Nu}-$ merous future directions exist. Firstly, we wish to generalise the results on uncoordinated attack and coordinated attack to arbitrary numbers of attacker nodes. Different leadership groups, and dissent topologies will also be explored. We also wish to investigate whether such straightforward strategies exist for more general topologies, and lastly, study strategies concerning social power for a subgroup of individuals.

\section{REFERENCES}

[1] N. E. Friedkin, "The Problem of Social Control and Coordination of Complex Systems in Sociology: A Look at the Community Cleavage Problem," IEEE Control Systems Magazine, vol. 35, no. 3, pp. 40-51, 2015.

[2] J. R. P. French Jr, “A Formal Theory of Social Power," Psychological Review, vol. 63, no. 3, pp. 181-194, 1956.

[3] M. H. DeGroot, "Reaching a Consensus," Journal of the American Statistical Association, vol. 69, no. 345, pp. 118-121, 1974.

[4] N. E. Friedkin, "A Formal Theory of Reflected Appraisals in the Evolution of Power," Administrative Science Quarterly, vol. 56, no. 4, pp. 501-529, 2011.

[5] D. Kempe, J. Kleinberg, and E. Tardos, "Maximizing the Spread of Influence through a Social Network," in Proceedings of the 9th ACM SIGKDD International Conference on Knowledge Discovery and Data Mining, 2003, pp. 137-146.

[6] P. Jia, A. MirTabatabaei, N. E. Friedkin, and F. Bullo, "Opinion Dynamics and the Evolution of Social Power in Influence Networks," SIAM Review, vol. 57, no. 3, pp. 367-397, 2015.

[7] Z. Xu, J. Liu, and T. Başar, "On a Modified DeGroot-Friedkin Model of Opinion Dynamics," in American Control Conference (ACC), Chicago, USA, July 2015, pp. 1047-1052.

[8] X. Chen, J. Liu, M.-A. Belabbas, Z. Xu, and T. Başar, "Distributed Evaluation and Convergence of Self-Appraisals in Social Networks," IEEE Transactions on Automatic Control, vol. 62, no. 1, pp. 291-304, 2017.

[9] J. R. P. French Jr and R. Snyder, "Leadership and Interpersonal Power," in Studies in Social Power, D. Cartwright, Ed. Research Center for Group Dynamics, Institute for Social Research, University of Michigan, 1959, ch. 8, pp. 118-149.

[10] V. S. Borkar, A. Karnik, J. Nair, and S. Nalli, "Manufacturing Consent," IEEE Transactions on Automatic Control, vol. 60, no. 1, pp. 104-117, 2015.

[11] C. D. Godsil and G. Royle, Algebraic Graph Theory. Springer: New York, 2001, vol. 207.

[12] M. Ye, J. Liu, B. D. O. Anderson, C. Yu, and T. Başar, "Evolution of Social Power in Social Networks with Dynamic Topology," 2017, arXiv:1705.09756 [cs.SI]. [Online]. Available: https://arxiv.org/abs/1705.09756

[13] lective Adjustment of Interpersonal Weights," 2017, arXiv:1703.03166 [cs:SI]. [Online]. Available: https://arxiv.org/abs/1703.03166 